\title{
Portable natural enhancement material resistivity meter : a prototype
}

\begin{abstract}
A new prototype of a portable natural enhancement material resistivity meter was successfully designed and constructed based on an existing soil resistivity meter. This portable natural enhancement material resistivity meter consists of four circuits, i.e. current generator, voltmeter, ammeter and display circuits, which these circuits needed to be compatible with each other to ensure the meter would be able to measure a correct resistivity of any material under test. Tremendous simulations were performed on each part of the four circuits to find the best suitable circuits that were compatible to each other to be constructed physically where a portable natural enhancement material resistivity meter could be made into its first prototype. Note that there are three types of material under tests involved in this work, i.e. soil, Bentonite and Zeolite, under three conditions, i.e. dry, mid-wet and highlywet. The prototype of a portable natural enhancement material resistivity meter is demonstrated to be perfectly working as the meter could be used to measure not only the soil resistivity but also resistivities of Bentonite and Zeolite too, which displayed higher resistivity values of dry specimens compared to the wet specimens.
\end{abstract}

Keyword: Natural enhancement material; Resistivity meter; Bentonite; Zeolite; Grounding system 